\title{
A Brief Review on Safety Strategies of Physical Human-robot Interaction
}

\author{
Nana Wang ${ }^{1, a}$, Yi Zeng ${ }^{1}$, Jie Geng ${ }^{2}$ \\ ${ }^{1}$ China National Institute of Standardization, 100191 Beijing, China \\ ${ }^{2}$ College of Quality and Safety Engineering, China Jiliang University, 310018 Hangzhou, China
}

\begin{abstract}
Nowadays, intelligent robotics are found in many places and always seem to be growing in complexity. The need to consider the human-robot interaction was further motivated by a growing number of collisions occurred among humans and robotics. The potential accidents need to be concerned and addressed urgently. This paper briefly reviewed some relevant researches on physical Human-robot Interactions, especially for the safety strategy issues. The suggestion to solve the physical Human-robot Interaction safety issues has been also proposed given to the review works.
\end{abstract}

\section{Introduction}

Nowadays, intelligent robotics are growing in complexity. Its application field has been extended from traditional industrial fields to medical treatment, logistics, housekeeping and other service fields. The need to consider the human-robot interaction was further motivated by a growing number of collisions occurred among humans and robotics. The potential accidents need to be concerned and addressed urgently.

It is understandable there has to have requirements and enhanced skills for humans and a demand of evolved robots in some more human-friendly machines. For a safe pHRI, safety is the basic requirement of a robot when working with humans. Human safety is generally guaranteed through slowing down or stopping motion when robot is approaching to human. Further, coexistence allows robotics sharing the workspace with humans. Collaboration is a special coexistence scenario that robotics interact with human directly. Safe collaboration has to be consistently guaranteed in a pHRI scenario [1].

The safety issue is always the first and core consideration should be guaranteed during the robot design phase. Safety strategies should be implemented. Current pHRI safety strategies cover pre-collision safety strategy and post-collision safety strategy [1]; or passive safety strategy and active safety strategy. Preventing pHRI collision is commonly used for active safety strategy; while reducing the collision forces during the special emergency scenario is a usual way for passive safety strategies in the robot design phase. Since there are various safety strategies for dealing with pHRI, some pHRI safety strategies are reviewed in the paper.

\section{Active (pre-collision) pHRI safety strategies}

\subsection{Safety strategy for pedestrian behavior prediction}

A probabilistic model was developed in [2] that can predict the behaviour of a pedestrian for mobile robots in the office and shopping mall HRI environment. The proposed safety strategy is for protecting mobile robots; however, it can be learned also for protecting human safety issue. This safety strategy was divided into two scenarios:

1) efficient mode can accurately predict the pedestrian behaviours; a probability of collision for human and robot can be estimated.

2) safety mode cannot support the prediction of the pedestrian behaviours, and the robot must do actions with a certain level of the safety emphasis.

In detailed modelling, the parameters refer to a set of behaviour patterns $\mathrm{q}$, positions $\mathrm{s}$, output directions $\mathrm{o}$, position output distributions aq(S), and direction output distributions $\mathrm{bq}(\mathrm{s}, \mathrm{o})$. Then, the robot will learn these model parameters through collecting behaviour data, classifying the data; and determining the output distributions of positions and directions. In the end, the trajectory will be generated to support making decision of different modes. For each mode, the different ranges of warning areas are also introduced to predict the collision risk, and the velocity control has been used to achieve these two safety strategies.

\subsection{Pre-collision HRI safety strategy}

Pre-collision safety strategies are generally dealing with safety at different time horizons. They have three

\footnotetext{
a Corresponding author: wangnn@cnis.gov.cn
} 
components [3]:

-Safe Planning (long-term safety) is a pathway planning with safety criteria. The method aims to place the robot in a better position when human is approaching to avoid unanticipated safety events. The planning module uses search strategies, like the best-first planning approach, or randomized planning. The method planned the safest path by searching contiguous regions without obstacles. It also leads to the goal and minimize a measure of danger criterion.

-Trajectory Scaling (medium term safety) is the path planning method that generates the velocity and acceleration profile to be followed along the path under the pHRI context. With an estimation of the danger from dynamic factors, it provides a relative safety path planning result.

-Reactive Control (short-term safety) is the key element of the safety module that estimates the danger level with danger index. With the calculated danger index, the virtual force to push the robot away from the human is generated.

Meanwhile, user monitoring technology is an alternative method sensing users' position and reactions. The information is used as inputs to the various safety modules.

\subsection{Learning-based safety strategy}

Learning-based safety strategy is initially designed for a robotic manipulator operating in an unstructured pHRI environment [4]. During reproduction,

- Kinematic Redundancy of the robot regulates the dynamic movement when the robot generates an infinite number of inverse control strategies.

- Task Redundancy regulates the stiffness of the robot when the task can be achieved through an infinite number of solutions.

After having several observations of a similar task, the robot creates a skill simulation model. The model considered the variations and correlations observed along the movement. If a part of a movement was consistent observed in different trials, the part should probably be reproduced in this specific manner.

\subsection{Withdrawal strategy for human safety}

Withdrawal strategy for human safety can be used when human and robot are approaching to each other in a very close range. The strategy aims to enlarge the distance from the robot to the human. Virtual force model regulates the end-effector velocity so that the robot can move away from human and meanwhile go to a nearest parking position [5].

\subsection{Constraint-based safety strategy}

Designing task-consistent collision avoidance strategies [6] productively combined task execution and safety actions in the HRI environment. Constraints affecting the task are concerned in the HRI safety strategy. Here, tasks' constraints are consistent and there is not necessary to have prioritization. Three task-oriented classifications (hard constraints, skill constraints, and soft constraints) support the exploitation of possible constraints redundancy while preserving the final task execution and completion. Moreover, unconstrained velocities can be exploited to perform evasive motions. Evasive motions aim to minimize the level of danger in HRI. For this, the danger field is introduced for assessing danger in HRI. Such measure concerns robotics as danger sources in its pHRI workspace. Both the distance and velocity between the human and robotics have to be predicted. The value of danger filed is computed and a state machine template to manage velocity constraints then will be proposed.

An integrated framework for social mapping [7] allowed robots to navigate in a pHRI environment. It demonstrates socially behaviour at the acceptable level. particularly one of "comfort, naturalness, sociability" responds the performed action by detecting the social map.

\section{Passive (post-collision) pHRI safety strategies}

Past research works also investigated the impacts of robotics from perspectives of realistic threats to human jobs, resources and safety, identity threats to human identity and distinctiveness, attitude toward robots, and support for robotics [8]. Participants were asked to watch videos of supposedly robotics and perceived that robots in general will be a significant threatening source to humans in future.

A new approach of human robot interaction (Human Hybrid Robot - HHR) [9] combined human and technical elements to supporting manual assembly tasks by taking advantages of individual abilities and technical benefits.

Assembly stations where human-robot collaborative tasks are carried out was designed [10]. Both passive and active pHRI safety strategies were combined to apply to guarantee the human safety and the overall system's productivity. A lot of clearance for robots has been required for fenceless separation monitoring. However, requirements for close cooperation of human and robotics are reduced in the collaborative and cooperative context. Here, the human is not constrained. The cooperation requires far more sensing technics for collision detection and collision avoidance. Regarding user's acceptance, operators feel more comfortable when they are aware of the underlying safety functionalities.

The requirements where humans and robots collaborate in an assembly process were studied in [11]. The robots assist working people in the assembly process. The requirements in terms of different aged operators in an ergonomic workplace are identified in line. The study also indicated that although "the corresponding technologies are already available, appropriate safety standards to ensure occupational safety are missing". The missing safety standards to response pHRI context especially focusing on the collaborative scenario is a main barrier to direct introduce robots into the pHRI working context.

A method for improving human performance has been 
introduced in [12]. The overall challenges are positively influenced by the method. The results lead to an enhanced pHRI experience.

A method presented by [13] is a systematic approach successfully in research projects. The method adapted a hazard identification technique, HAZOP (Hazard Operability), coupled with UML (Unified Modeling Language).

In the early design phase of a robot, planning for safety to identify the potential HRI hazards received less attention than techniques. Planning for safety is an important way to reduce potential hazards under the HRI context. Safety criteria need to identify in the planning phase [3]. With the safe planning and designing, the robot will improve the performance as well [14]. Some robotic standards included the commonly used industrial risk assessment methods to guarantee the pHRI safety. However, these standards [15-16] rarely meet the requirements for dealing with close pHRI scenarios. Appropriate policies for controlling the relevant risks and associated control strategies need to be examined [17-18].

\section{Conclusion}

Safety is the most essential requirement of human-robot interaction. In addition to taking hardware and software measures to ensure the objective attributes of robot safety. It is also necessary to further study the subjective attributes of human feelings and psychology in the process of human-robot interaction, so as to evaluate the safety problems more objectively and accurately.

\section{References}

1. Heinzmann, J., \& Zelinsky, A. (2003). Quantitative safety guarantees for physical human-robot interaction. The International Journal of Robotics Research, 22(7-8), 479-504.

2. Tamura, Y., Le, P. D., Hitomi, K., \& Chandrasiri, N. P. (2012). Development of pedestrian behavior model taking account of intention. IEEE/RSJ International Conference on Intelligent Robots \& Systems.

3. Kulić, D., \& Croft, E. (2007). Pre-collision safety strategies for human-robot interaction. Autonomous Robots, 22(2), 149-164.

4. Calinon, S., D'halluin, F., Sauser, E. L., Caldwell, D. G., \& Billard, A. G. (2010). Learning and reproduction of gestures by imitation. IEEE Robotics \& Automation Magazine, 17(2), 44-54.

5. Ricardez, G. A. G., Yamaguchi, A., Takamatsu, J., \& Ogasawara, T. (2013, November). Withdrawal strategy for human safety based on a virtual force model. In Intelligent Robots and Systems (IROS),
2013 IEEE/RSJ International Conference on (pp. 1119-1124). IEEE.

6. Ceriani, N. M., Zanchettin, A. M., Rocco, P., Stolt, A., \& Robertsson, A. (2013, November). A constraint-based strategy for task-consistent safe human-robot interaction. In Intelligent Robots and Systems (IROS), 2013 IEEE/RSJ International Conference on (pp. 4630-4635). IEEE.

7. Charalampous, K., Kostavelis, I., \& Gasteratos, A. (2016). Robot navigation in large-scale social maps: An action recognition approach. Expert Systems with Applications, 66, 261-273.

8. Złotowski, J., Yogeeswaran, K., \& Bartneck, C. (2017). Can we control it? Autonomous robots threaten human identity, uniqueness, safety, and resources. International Journal of Human-Computer Studies, 100, 48-54.

9. Weidner, R., Kong, N., \& Wulfsberg, J. P. (2013). Human Hybrid Robot: a new concept for supporting manual assembly tasks. Production Engineering, 7(6), 675-684.

10. Michalos, G., Makris, S., Tsarouchi, P., Guasch, T., Kontovrakis, D., \& Chryssolouris, G. (2015). Design considerations for safe human-robot collaborative workplaces. Procedia CIrP, 37, 248-253.

11. Faber, M., Bützler, J., \& Schlick, C. M. (2015). Human-robot cooperation in future production systems: analysis of requirements for designing an ergonomic work system. Procedia Manufacturing, 3, 510-517.

12. Guerrero, C. R., Marinero, J. C. F., Turiel, J. P., \& Muñoz, V. (2013). Using "human state aware" robots to enhance physical human-robot interaction in a cooperative scenario. Computer methods and programs in biomedicine, 112(2), 250-259.

13. Guiochet, J. (2016). Hazard analysis of human-robot interactions with HAZOP-UML. Safety science, 84, 225-237.

14. Macfarlane, S., \& Croft, E. A. (2003). Jerk-bounded manipulator trajectory planning: design for real-time applications. IEEE Transactions on Robotics and Automation, 19(1), 42-52.

15. ISO 10218:1992. Manipulating industrial robots Safety manual.

16. Gaskill, S. P., \& Went, S. R. G. (1996). Safety issues in modern applications of robots. Reliability Engineering \& System Safety, 53(3), 301-307.

17. Calinon, S., D'halluin, F., Sauser, E. L., Caldwell, D. G., \& Billard, A. G. (2010). Learning and reproduction of gestures by imitation. IEEE Robotics \& Automation Magazine, 17(2), 44-54.

18. Gaskill, S. P., \& Went, S. R. G. (1996). Safety issues in modern applications of robots. Reliability Engineering \& System Safety, 53(3), 301-307. 